\title{
Numerical Simulation of Fluid Structure Coupling of Pile in Deep Water
}

\author{
Yan Wenyue ${ }^{1, \text { a }}$,Lv Fengwu ${ }^{1, b}$ \\ ${ }^{1}$ Department of Building Engineering,College of Civil Engineering,Tongji University,1239 Siping \\ Road,Shanghai 200092,P.R.China \\ a1432043ywy@tongji.edu.cn,, ${ }_{\text {ffengwur@tongji.edu.cn }}$
}

Keywords:the two-way coupling, current force, shelter coefficient of piles transverse, coefficient of piles

Abstract.In this paper, the two-way coupling of flow and pile is simulated by using ANSYS Workbench. A comparison between the results of current force in one-way coupling, two-way coupling and code method has been made, proving a reference for the calculation of water flow in engineering. Four sets of influence coefficients under the circumstances of three parallel piles and three tandem piles were simulated respectively.And a comparison of transverse coefficient among numerical simulation and code has been made. It is found that the shelter coefficient is not only influenced by the spacing of the piles but also by the water velocity, and the transverse coefficients the parallel piles have superposition effect.

\section{Introduction}

The trend of ocean development is towards deep waters. People have built many structures in deep waters in recent years, such as oil drilling platforms, offshore wind power, ports and bridges crossing seas and rivers. In deep water engineering, current force is one of the dominate load. However, the effect of flow force on the structure in water is generally considered as static force at the engineering calculation of present, so the effect of structure on current force is mainly reflected in the projected area of the vertical direction of flow, shape of piles, pile spacing and so on, but the effect of structure displacement and deformation on the flow is ignored. Although this calculation method is easy to use in engineering, it's not suitable for deep waters engineering. As the structure has a great influence on the flow of water in deep water because of the large deformation of the structure. It is significant to study the flow force of the structure in deep water, from the viewpoint of fluid structure interaction.

It's a tendency to study fluid structure coupling by means of numerical simulation, with rapid development of calculation. Liu Yongji [1] used ANSYS to analyze the ratio of static water depth to the piles depth, composite elastic modulus of pile, ratio of thickness of hollow pile to the external diameter and influences of those to the fluid structure coupling. Lei Fan[2] used ANSYS CFX to study the vibration performance of the underwater flexible structures and mechanism performance under loads like current force and earthquake load, from the viewpoint of fluid structure interaction. Li Chao [3] used ANSYS Workbench to make a fluid solid interaction analysis on hydraulic floating wave power device. ZuoShengrong[4] simulated the role of circular piers combined action of waves and uniform flow and analyzed the response of the piers.

Documents above mainly focus on numerical simulation of waves and fluid solid coupling between waves and simple structure model, but they seldom focus on coupling effect between water flow and structure, especially the two-way fluid structure coupling effect. In this paper, the interaction between water flow and steel pipe piles have been taken into consideration by 
establishing two-way fluid solid coupling models in ANSYS Workbench platform, and a study have been made about the transverse coefficient of three parallel piles and the shelter coefficient of three tandem piles.

\section{Theoretical Model and Control Equation}

Equation of Fluid Solid Coupled System Field. Fluid is assumed to be free viscous, compressible and small perturbations, and free liquid level to be small fluctuations, and solid is assumed to be linear elastic. So the linear elasticity structure and ideal compressible fluid constitute a linear fluid solid coupling problem, and the finite element analysis models can be mainly divided into two types: Displacement-Displacement form and Displacement-Potentials form. As the Displacement-Potentials form is more efficient, it is highly used in engineering now. It means that the equation of solid domain uses displacement $u_{i}$ to be basic unknown quantity, and the equation of fluid domain uses flow field pressure $p$ to be basic unknown quantity. The corresponding finite element expression format is called displacement-pressure $\left(u_{i}, p\right)$ scheme for fluid solid coupling analysis[5].

\section{Fluid Field}

(1) Fluid field equation:

$$
p_{, i i}-\frac{1}{c_{o}^{2}} \ddot{p}=0
$$

$p$ stands for fluid pressure, $c_{o}$ stands for sound velocity in fluid.

(2) Fluid boundary conditions

Rigid fixed boundary:

$$
\frac{\partial p}{\partial n_{f}}=0
$$

Free surface:

$$
\frac{\partial p}{\partial z}+\frac{1}{g} \ddot{p}=0
$$

\section{Solid Field}

(1)Solid field equation

$$
\sigma_{i j, j}+f_{i}=\rho_{s} \ddot{u}_{i}
$$

$\sigma_{i j}$ stands for solid stress component, $u$ stands for solid displacement component, $f_{i}$ stands for

solid volume force component, $\rho_{s}$ stands for solid mass density.

(2)Solid boundary condition

Force boundary condition 


$$
\sigma_{i j} n_{s j}=\bar{T}_{i}
$$

Displacement boundary condition

$$
u_{i}=\bar{u}_{i}
$$

$\bar{T}_{i}$ stands for known surface force component on solid, $\bar{u}_{i}$ stands for known displacement components on solids.

\section{Compatibility Conditions on the fluid solid coupling interface}

(1)kinematic condition: the normal velocity of the fluid solid interface should be continuous, which means

$$
v_{f n}=v_{f} \cdot n_{f}=v_{s} \cdot n_{f}=-v_{s} \cdot n_{s}=v_{s n}
$$

At the fluid solid interface, using the equation of motion of the fluid the formula can be rewritten as

$$
\frac{\partial p}{\partial n_{f}}+\rho_{f} \ddot{u} \cdot n_{f}=0
$$

$u$ means solid displacement vector, $\rho_{f}$ means fluid mass density.

(2)Dynamitic condition: normal stress should be continuous on the interface of fluid solid interface, which means

$$
\sigma_{i j} n_{s j}=\tau_{i j} n_{f j}=-\tau_{i j} n_{s j}
$$

$\tau_{i j}$ stands for component of fluid stress tensor. For free viscous fluid, $\tau_{i j}$ can be represents as

$$
\tau_{i j}=-p \delta_{i j}
$$

Taking the formula into formula(9), then gaining this formula

$$
\sigma_{i j} n_{s j}=p n_{s j}
$$

Turbulence Model.For the fluid structure coupling problem in deep water, fluid should be considered as turbulent fluid, thus turbulent equation need to be added additionally. In this paper, the standard $k-\varepsilon$ turbulence model is used.

Equation of fluctuating kinetic energy $k$ :

$$
\frac{\partial(\rho k)}{\partial t}+\nabla \cdot(\rho \boldsymbol{u} k)=\nabla \cdot\left[\left(\mu+\frac{\mu_{t}}{\sigma_{k}}\right) \nabla k\right]+P_{k}-\rho \varepsilon+S_{k}
$$

Equation of turbulent dissipation rate $\varepsilon$ :

$$
\frac{\partial(\rho \varepsilon)}{\partial t}+\nabla \cdot(\rho \boldsymbol{u} \varepsilon)=\nabla \cdot\left[\left(\mu+\frac{\mu_{t}}{\sigma_{\varepsilon}}\right) \nabla \varepsilon\right]+\frac{\varepsilon}{k}\left(C_{\varepsilon 1} P_{k}-C_{\varepsilon 2} \rho \varepsilon\right)+S_{\varepsilon}
$$

Eddy viscosity $\mu_{t}$ :

$$
\mu_{t}=C_{\mu} \rho \frac{k^{2}}{\varepsilon}
$$




$$
P_{k}=\mu_{t} \nabla \boldsymbol{u} \cdot\left(\nabla \boldsymbol{u}+\nabla \boldsymbol{u}^{T}\right)-\frac{2}{3} \nabla \cdot \boldsymbol{u}\left(3 \mu_{t} \nabla \cdot \boldsymbol{u}+\rho k\right)
$$

$k$ stands for fluctuating kinetic energy, $\varepsilon$ stands for turbulent dissipation rate, $S$ stands for custom source terms.

In $k-\varepsilon$ turbulence model, $C_{\mu}, \sigma_{k}, \sigma_{\varepsilon}, C_{\varepsilon 1}, C_{\varepsilon 2}$ are empirical constants, whose value can refer to documentation [6].

\section{Numerical Solution Method}

The partial differential equations of fluid structure coupling shown above are quite complicated. Coupling with the complexity of boundary condition of fluid domain and solid domain, it's difficult to get the analytical solution of the partial differential equation shown above. However, it is common to use numerical analysis method by computer to solve the problem of solving differential equations with a given boundary condition. Commonly used numerical analysis methods are Finite Difference Method(FDM),Finite Element Method(FEM), Finite Volume Method(FVM). This paper use FEM in ANSYS Structure to solve the equations of solid domain, while using FVM in ANSYS CFX to solve the equations of fluid domain.

Displacement-Pressure form is used as the finite element analysis model in this paper. The displacement of solid domain transmit to the fluid field, considering the nodal displacement as boundary condition, the equation that use the pressure of the flow field as the unknown variable can be solved. And then flow field transmit the boundary pressure to the solid domain, which consider nodal displacement as the unknown quantity of the basic equation. After solving the nodal displacement, the nodal displacement is transmitted to the fluid field, which will become the boundary condition of fluid field, and then circulate until converge.

This paper use ANSYS Workbench to carry out numerical simulation of fluid solid coupling. Workbench use CFX and Structure to solve the equation of fluid and solid in the same time step, and then use MFX procedure of ANSYS to exchange the data on the fluid solid coupling surface between the solid and fluid domains. The solid and fluid fields are calculated according to the updated boundary conditions. Its specific realization thought is shown as the Fig. 1. 


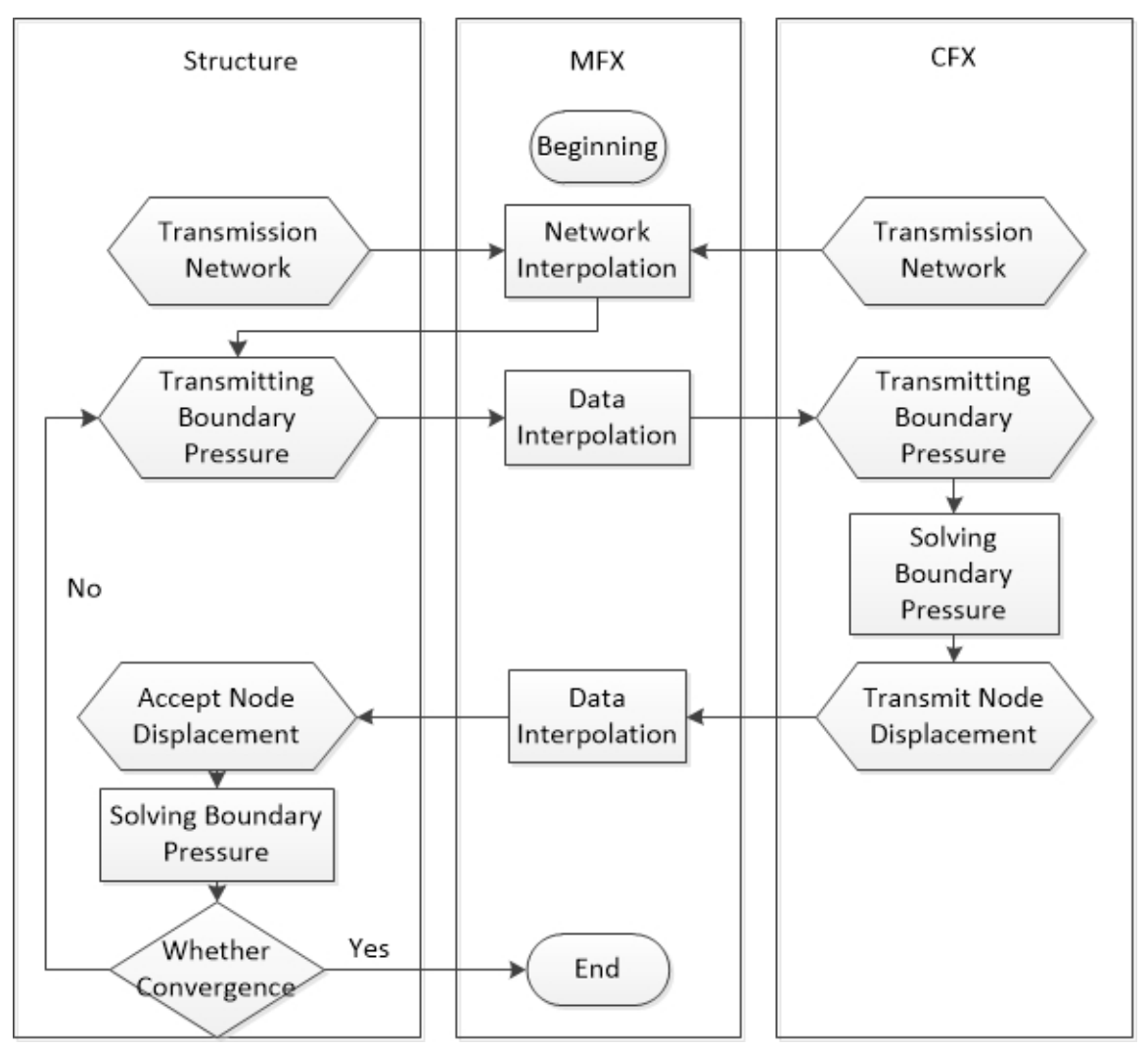

Fig. 1 the Realization of Fluid Structure Coupling

The coupling iteration number is controlled by the convergence accuracy. And it is taken as the convergence control criterion when RMS less than 1e-4 in this paper.

\section{Case Analysis}

This paper takes a steel pipe pile foundation of a construction platform in the mouth of Yangtze River as an example to analysis fluid solid coupling. And the $25 \mathrm{~m}$-high steel pipe pile embedded in river bed, whose diameter is $2.54 \mathrm{~m}$ and thickness is $0.06 \mathrm{~m}$. And the steel pipe pile is in the $25 \mathrm{~m}$-deep water flow whose rate is $2 \mathrm{~m} / \mathrm{s}$.

\section{Single Pile Analysis.}

(1)Distribution of Fluid Pressure.The basic law of the distribution of pressure in water flow can get from Figure 2 and Figure 3.

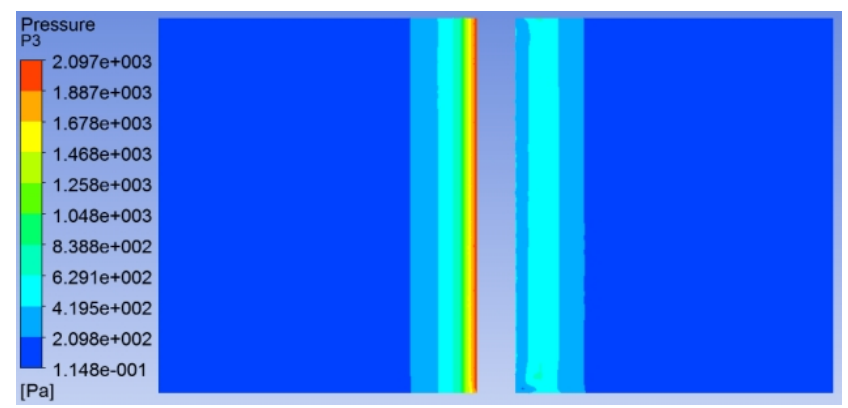

Fig. 2 Fluid Pressure Cloud of Longitudinal Symmetry Plane

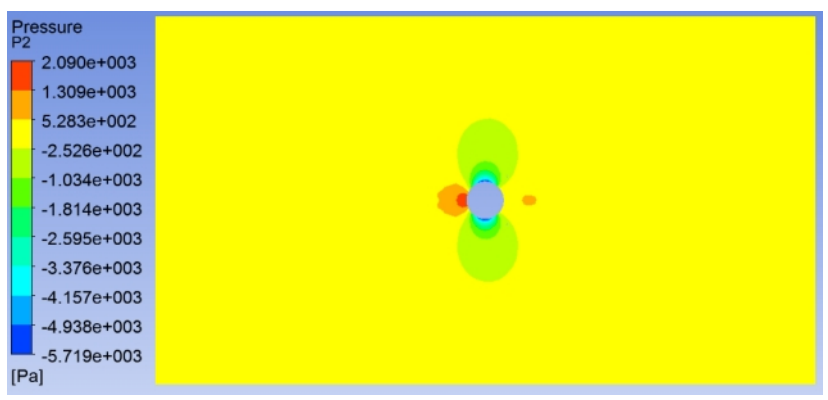

Fig. 3 Fluid Pressure Cloud of 12.5m- depth plane

(2)Comparison of Stress Calculation Results of Pile. Making a comparison of the stress at the bottom of the pile, the results of three calculation methods are showed as table 1. What's more, the 
influence of the solid deformation to the water field is neglected in the one-way coupling, and the current force is regarded as static force in the code method.

Table 1: Comparison of Stress of Pile

\begin{tabular}{|c|c|c|c|}
\hline Computing Method & one-way coupling & two-way coupling & Code method \\
\hline Stress of Pile $\sigma[\mathrm{MPa}]$ & 2.99 & 3.14 & 5.09 \\
\hline
\end{tabular}

From table 1 it can be known that the calculating results of the one-way coupling and two-way coupling are very close to, whose difference is only $4.8 \%$; while the results of code method is larger than that of two-way coupling by $62.1 \%$.

It shows that the stiffness of steel pipe pile is large, and the deformation of the pile is not significant, and the code method of current force is partial safety.

The Shelter Influence Analysis of Tandem Pile.L stands for spacing of pile and D is the diameter of the steel pipe pile. Setting the shelter coefficient of flow is :

$$
m_{i}=\frac{F_{i}}{F_{s i}}=\frac{\sigma_{i}}{\sigma_{s i}}
$$

$F_{i}$ is the current force of steel pipe pile of No.i; $F_{s i}$ is the current force of isolated steel pipe pile; $\sigma_{i}$ is the maximum stress of steel pipe pile of No.i,and that is $3.48 \mathrm{MPa} ; \sigma_{s i}$ is the maximum stress of isolated steel pipe pile.

By Taking $\mathrm{L} / \mathrm{D}=2,3,4,5$, the shelter coefficients of isometric three piles by numerical simulation can be seen in Figure 4.

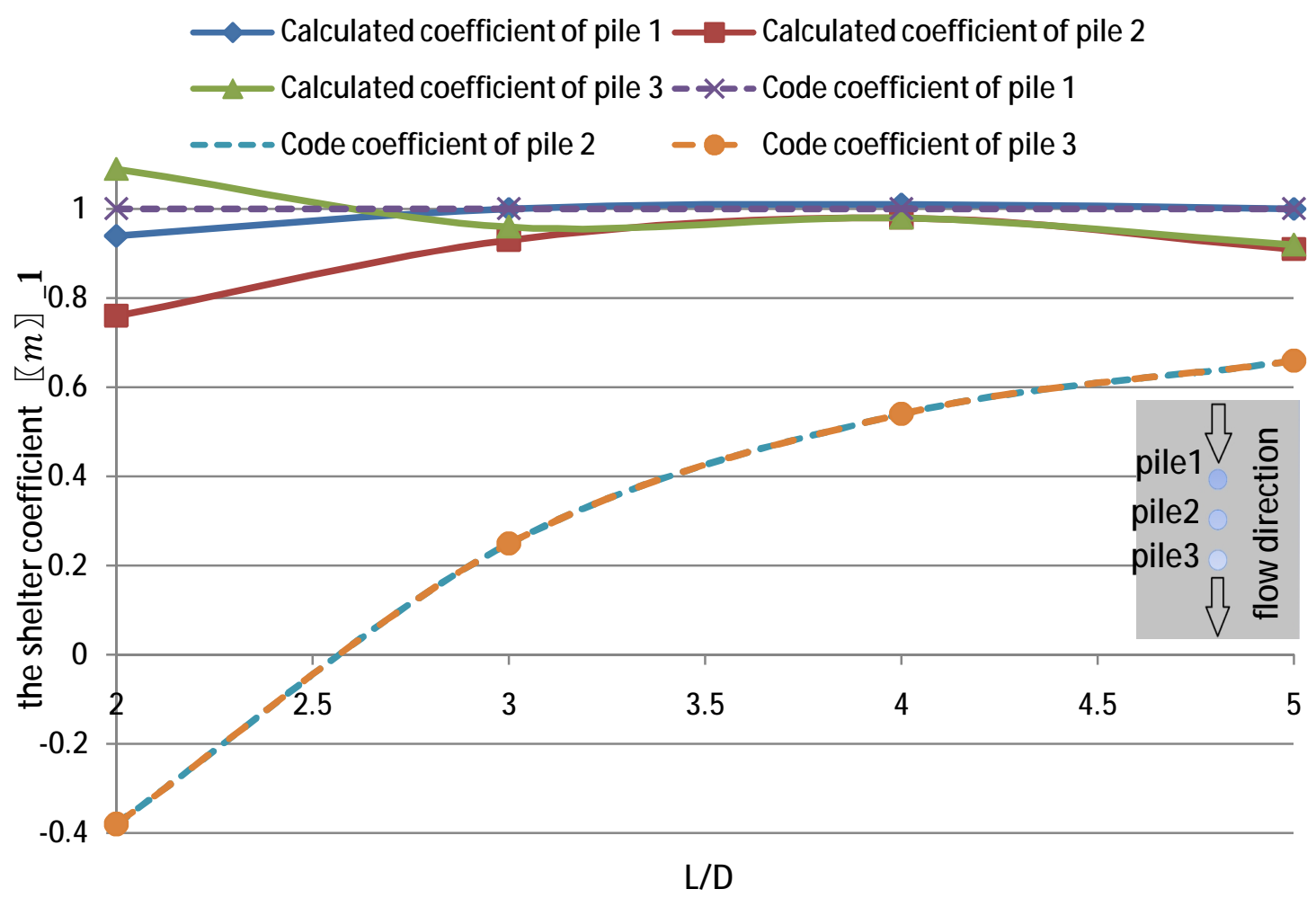

Fig. 4 the Comparison of Shelter Coefficient

The numerical simulation results of the front row pile (Pile 1) are very close to those of the code, while the coefficients of shelter to back row piles (Pile 2 and Pile 3)are quite different with those of the code.In general, the influence of shelter to back row pile is far less than that of the front row 
pile. What's more, the flow may form negative pressure zone between the piles, and then the back row pile is subject to tension by flow, so that the negative coefficient is formed. At last, excepting for the pile spacing, the code method of shelter coefficient should be further refined, such as velocity of the water flow and so on.

The Transverse Influence Analysis of Parallel Piles.Setting the transverse coefficient of flow is:

$$
m_{i}^{\prime}=\frac{\sigma_{i}}{\sigma_{s i}}(17)
$$

By Taking $\mathrm{L} / \mathrm{D}=2,3,4,5$,the transverse coefficient of isometric three piles by numerical simulation can be seen in Figure 5.

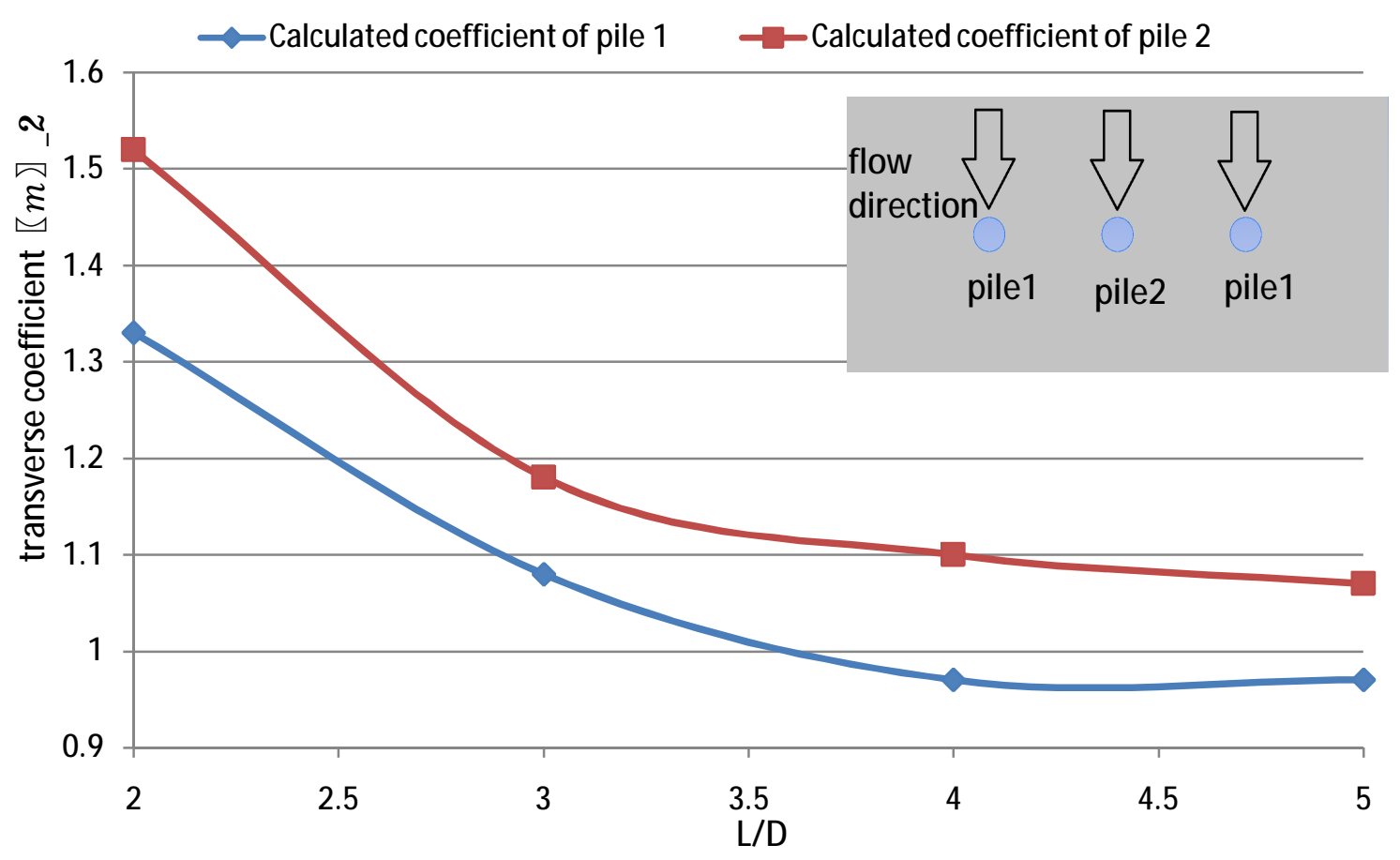

Fig. 5 Transverse Coefficient of Three Parallel Piles

Figure 5 show that the pile spacing is smaller, the transverse coefficient of flow is greater, that is the lateral influence is greater.When the pile spacing is $5 \mathrm{D}$, the transverse coefficient is close to 1 in the background of the research in this paper, so the transverse coefficient of flow can be ignored.

\section{Conclusions}

In this paper, the two-way coupling of uniform flow and pile is simulated and analyzed by using ANSYS Workbench platform, and the following conclusions are obtained:

(1) The effect of the two-way coupling of pile and water depends on the stiffness of pile. When the stiffness of pile is large,the results of one-way coupling and two-way coupling are close to.Considering the two-way coupling consumes great computational resources, it is recommended to calculate current force by one-way coupling. What's more, the code method of current force is partial safety, which considering the current force as static force.

(2) It is found that the shelter coefficient is generally less than 1, and the shelter influence of front row pile is far less than that of the back row pile.

(3) The shelter coefficient is not only influenced by the spacing of the piles but also by other factors,such as the velocity of the water, pile shape... 
(4) The transverse influences of piles are obvious, when the pile spacing is small. The transverse coefficients are generally greater than 1 , and the transverse influence of center pile is far larger than that of the side piles.

\section{References}

[1] Liu Yongji, Song Jianyu, Zhu Lianghu. Influence on Pile Frequency Characteristic by Fluid-Structure Interaction Effect [J]. Harbor Engineering, 2013, 50 (1): 22-24.

[2] Ray fan. Dynamic Response Research of Submarine Flexible Structures with Fluid-Solid Interaction [D]. Wuhan University of Technology, 2011

[3] Li Chao. Fluid Structure Interaction Analysis of the Full Hydraulic Floating Wave Power Generator [D]. Shandong University master's degree thesis, 2012

[4] ZuoShengrong. Study on Wave Effect of Bridge Pier ofCrossing Bridge in Deep Water of Sea [D]. Wuhan University of Technology, 2013

[5] Zhu Yanrong. Ocean Engineering Wave Mechanics [M].The first edition. Tianjin: Tianjin University press, 1998

[6] Liu Zheng Xian, Song Baojun, valley Chuangang,.Numerical Study of High Order Anisotropic $k-\varepsilon$ Models and Its Coefficients.Journal of Engineering Thermal Physics, 1996. 17 (Suppl): 31-34.

[7] The first and second Harbor Engineering Survey \& Design Institute Co., Ltd.LoadCode for Harbour Engineering [M]. The first edition. Beijing: China Communications Press, December 2010. 